\title{
Usefulness of Clipless Laparoscopic Division of Superior Right Colic Vein for Right Side Transverse Colon Cancer
}

\author{
Hidejiro Kawahara ${ }^{1 *}$, Nobuo Omura², Tadashi Akiba'
}

'Department of Surgery, Kashiwa Hospital, Jikei University School of Medicine, Chiba, Japan 2Department of Surgery, Nishisaitama-chuo national Hospital, Saitama, Japan

\section{ABSTRACT}

Background: Clips are commonly used to divide vessels in laparoscopic surgery. However, branches of the superior mesenteric vein (SMV), including the superior right colic vein (SRCV), are narrow, and the clips are too large to be used for these veins. During surgery, there is concern about the risk of bleeding due to vascular injury, as well as when detaching the clips after the procedure.

Patients and Methods: From January 2014 to December 2016, six patients with right side transverse colon cancer underwent clipless laparoscopic surgery were enrolled in this study. After skeletonizing around the roots of the SRCVs, they were divided at their roots only by LigaSure, without the use of clips.

Results: The mean operative time was 136 min (range 114-160), the mean operative blood loss was less than $10 \mathrm{ml}$, and no bleeding at the stump of the SRCV was confirmed during surgery. The mean length of hospitalization after surgery was 10 days, and no complications were encountered after surgery. No recurrence was found more than three years after surgery.

Conclusion: Clipless laparoscopic division of the SRCV is a useful procedure for patients with right side transverse colon cancer.

Key words: clipless laparoscopic surgery, partial transverse colectomy, LigaSure

\section{INTRODUCTION}

Currently, laparoscopic surgery is applied for the treatment of a variety of diseases. Here, we report total colectomy via a clipless laparoscopic surgical procedure with a single incision (1). There are few reports on clipless laparoscopic partial colectomy for patients with right side transverse colon cancer. Clips are commonly used to divide vessels in laparoscopic surgery. However, the branches of the superior mesenteric vein (SMV), including the superior right colic vein (SRCV), are narrow, and the clips are too large to be used for these veins. During surgery, there is concern about the risk of bleeding due to vascular injury, as well as when detaching the clips after the procedure; a clipless procedure reduces these concerns. We performed a novel procedure: a clipless laparoscopic partial colectomy for right side transverse colon cancer.

\author{
Corresponding author: \\ Hidejiro Kawahara, MD, PhD \\ Department of Surgery \\ Kashiwa Hospital, Jikei University \\ School of Medicine \\ 163-1 Kashiwashita, Kashiwashi, \\ Chiba 277-8567, Japan \\ Tel: +81471641111 Ext. 3421 \\ Fax: +81471633488 \\ E-mail: kawahide@outlook.jp \\ ORCID ID: 0000-0002-8618-1556
}

Received: 04.11.2020

Accepted: 12.01.2021

Copyright (1) Celsius Publishing House www.sgo-iasgo.com 


\section{MATERIALS AND METHODS}

From January 2014 to December 2016, six patients with right side transverse colon cancer underwent clipless laparoscopic surgery, were retrospectively registered. The cohort consisted of two patients with stage I and four patients with stage III colon cancer (table 1). In this procedure, dissection around the vessels was performed only with a sealing device, LigaSure, without the use of clips. SRCV, the middle colic vein (MCV), and the middle colic artery (MCA) were also divided at their roots only by LigaSure, without the use of clips.

The Ethics Committee for Biomedical Research of the Jikei Institutional Review Board approved the protocol [30-344 (9365)], and all patients or their family members provided written informed consent to participate.

\section{SURGICAL TECHNIQUES}

First, the greater omentum was divided by an approach with five ports. Then, the division was extended laterally to hepatic flexures of the colon. A window in the mesocolon was created from the descending to the horizontal portion of the duodenum. The SRCV, located on the patients' left side of the window, was detected and skeletonized around its root (fig. 1). After the skeletonizing, they were divided at their root by a single sealing of LigaSure without clips (fig. 2). After a partial transverse colectomy following the dissection of the middle colic vein and colic artery, intestinal reconstruction was performed with a stapler to create a functional end-to-end anastomosis.

Table 1 - Characteristics of the patients

\begin{tabular}{lc}
\hline Variable & $\mathbf{n = 6}$ \\
\hline Age (years) & $71.3(46-81)$ \\
\hline Gender & $2(33)$ \\
$\quad$ Male & $4(67)$ \\
$\quad$ Female & $136.0(114-160)$ \\
\hline Mean surgical time (min) & $10>$ \\
\hdashline Mean blood loss (ml) & 10 \\
Mean postoperative hospital stay (days) & $2(33)$ \\
\hdashline Stage & $0(0)$ \\
I & $3(50)$ \\
II & $1(17)$ \\
III a &
\end{tabular}

The data are presented as mean (range) or as $\mathrm{n}(\%)$

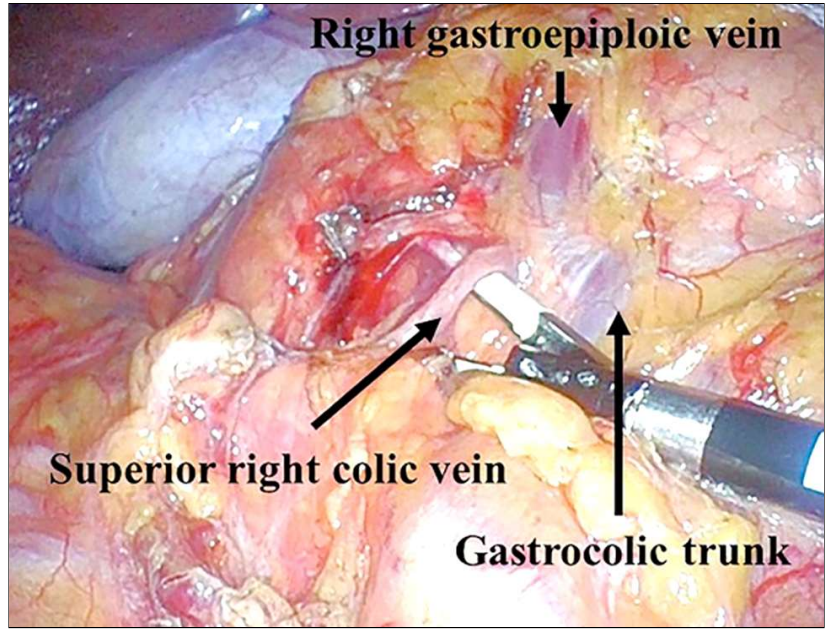

Figure 1 - Surgical view around the gastrocolic trunk of Henle before the division. The superior right colic vein was detected and skeletonized around its root

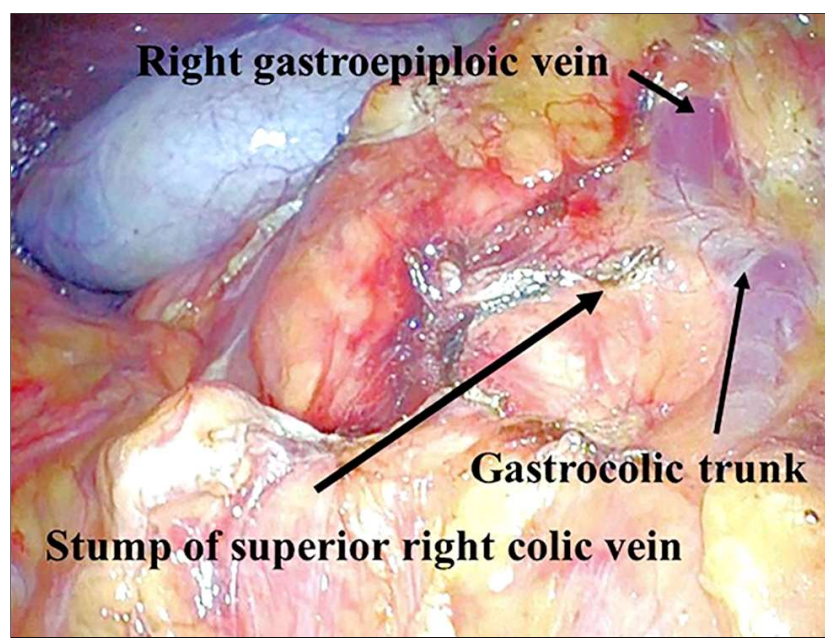

Figure 2 - Surgical view around the gastrocolic trunk of Henle after the division. No bleeding at the stump of the superior right colic vein was confirmed during surgery.

\section{RESULTS}

The mean operative time was 136 min (range 114160 ), the mean operative blood loss was less than 10 $\mathrm{ml}$, and no bleeding at the stump of the SRCV were confirmed during surgery. The mean length of hospitalization after surgery was 10 days, and no complications were encountered after surgery. No recurrence was found more than three years after surgery.

\section{DISCUSSION}

The risk of bleeding in the SMV during laparoscopic right hemicolectomy or partial transverse colectomy has increased since the introduction of D3 lymph node dissection (2). The main cause of bleeding is the 
laparoscopic dissection of the gastrocolic trunk of Henle (3). Many variations have been identified in the formation and drainage routes of other venous colic tributaries of the SMV (4). In a right hemicolectomy and partial transverse colectomy for patients with colon cancer, these vessels are divided at their roots with D3 lymph node dissection (5-7). Clips have been commonly used to divide vessels in laparoscopic surgery. However, the branches of the SMV, including the SRCV, are narrow, and the clips are too large for these veins. During surgery, there is concern about the risk of bleeding due to vascular injury, as well as when detaching the clips after the procedure.

We reported total colectomy via a clipless laparoscopic surgical procedure with a single incision surgery (1). In this procedure, the MCA and MCV were divided slightly away from their roots by a double sealing of LigaSure with incomplete skeletonization around them. No bleeding was confirmed at the stumps of vessels. In the present study, the MCA and MCV were divided at their roots by a double sealing of LigaSure with complete skeletonization around their roots. Branches of the SMV, including the SRCV, were divided at their roots by a single sealing of LigaSure. No bleeding was confirmed at the stumps of vessels.

The division of vessels with clips requires a long length of skeletonized vessels because vessels have to be divided between the proximal and distal clips. Since clipless vascular division requires a shorter length of skeletonized vessels compared to those needed for the procedure with clips, clipless vascular division seems feasible and safe.

Clipless laparoscopic surgery is actively applied for cholecystectomy however there are few reports on clipless laparoscopic colectomy for patients with colorectal diseases (8-10).

Long-term follow-up and large-scale evaluation are necessary, but clipless laparoscopic surgery seems to be a useful procedure for patients with right side transverse colon cancer.

\section{Ethics approval and consent to participate}

The Ethics Committee for Biomedical Research of the Jikei Institutional Review Board approved the protocol [30-344 (9365)], and all patients or their family members provided written informed consent to participate.

\section{Consent for publication}

The written consent to publish images or other personal or clinical details of participants was obtained from the patient.

\section{Availability of data and materials}

All data generated or analysed during this study are included in this published article.

\section{Competing interests}

The authors declare no competing interests.

\section{REFERENCES}

1. Kawahara H, Watanabe $\mathrm{K}$, Tomoda M, Enomoto H, Akiba T, Yanaga K. Single-incision Clipless Laparoscopic Total Colectomy. Hepatogastroenterology. 2014;61:453-5.

2. Watanabe T, Itabashi M, Shimada Y, Tanaka S, Ito Y, Ajioka Y, et al. Japanese Society for Cancer of the Colon and Rectum (JSCCR) Guidelines 2010 for the Treatment of Colorectal Cancer. Int J Clin Oncol. 2012:17:1-29.

3. Mike M, Kano N. Laparoscopic Surgery for Colon Cancer: A Review of the Fascial Composition of the Abdominal Cavity. Surg Today. 2015;45:129-39.

4. Gillot C, Hureau J, Aaron C, Martini R, Thaler G, Michels. The superior mesenteric vein, anatomy and surgical study of 81 subjects. J Int Coll Surg 1964;41:339-69.

5. Kuzu MA, Ismail E, Celik S, Sahin MF, Güner MA, Hohenberger W, et al. Variations in the Vascular Anatomy of the Right Colon and Implications for Right-Sided Colon Surgery. Dis Colon Rectum. 2017;60:290-8.

6. Garcia-Granero A, Sánchez-Guillén L, Frasson M, Sancho Muriel J, Alvarez Sarrado E, Fletcher-Sanfeliu D, et al. How to Reduce the Superior Mesenteric Vein Bleeding Risk During Laparoscopic Right Hemicolectomy. Int J Colorectal Dis. 2018;33:235-9.

7. Ye K, Lin J, Sun Y, Wu Y, Xu J, He S. Variation and Treatment of Vessels in Laparoscopic Right Hemicolectomy. Surg Endosc. 2018;32:1583-4.

8. Swanstrom LL. "Clipless" cholecystectomy: evolution marches on, even for lap chole. World J Surg. 2011;35:824-5

9. Wills E, Crawford G. Clipless versus conventional laparoscopic cholecystectomy. J Laparoendosc Adv Surg Tech A. 2013;23:237-9.

10. Ramos AC, Ramos MG, Galvăo-Neto Mdos P, Marins J, Bastos EL Zundel N. Total clipless cholecystectomy by means of harmonic sealing. Arq Bras Cir Dig. 2015;28:53-6. 